\title{
Extension of the Deal-Grove Oxidation Model to Include the Effects of Nitrogen
}

\author{
Sima Dimitrijev, Member, IEEE, H. Barry Harrison, and Denis Sweatman
}

\begin{abstract}
Indications are that very thin dielectrics needed for future generations of integrated circuits will be in a form of nitrogen-modified oxide. A significant amount of experimental data on growth kinetics for oxides grown/nitrided in $\mathrm{N}_{2} \mathrm{O}$ has been gathered. It appears that nitrogen neutralizes growth sites at the oxide-silicon interface, which significantly slows down the oxidation process when $\mathrm{N}_{2} \mathrm{O}$ is used as an oxidizing ambient. In this paper, the classic Deal-Grove formulation is extended to include the concentration of the growth sites. Also, the continuity equation applied to the growth sites is used to determine the concentration of the growth sites. This model has been incorporated into TMA SUPREM-3 process simulator, and the model parameters calibrated with available experimental data. This provides not only a tool needed for process simulation, but also a better understanding of nitrogen modified oxide films.
\end{abstract}

\section{INTRODUCTION}

$\mathbf{T}$ $\mathrm{HE}$ quality of thermally grown $\mathrm{SiO}_{2}$ films enabled fabrication of MOSFET's in 1960 [1]. The process of thermal oxidation has been the cornerstone of silicon technology ever since. It has been evident for some time, however, that pure $\mathrm{SiO}_{2}$ does not have the physical and electrical properties necessary to sustain the continuing reduction in device dimensions [2]. There is substantial evidence that incorporation of nitrogen, in one form or another, significantly improves the properties of the ultra-thin oxide films. In particular, oxides grown or nitrided in $\mathrm{N}_{2} \mathrm{O}$ have shown significant improvements over their pure oxide counterparts [3]-[8]. We have obtained results [9]-[11] indicating that oxides grown/nitrided in $N O$ could provide even better electrical and physical properties. It is very likely that a solution for the gate dielectrics needed for future generations of integrated circuits will be found in some kind of nitrogen-modified oxide.

The current understanding and modeling of the thermal oxidation process is based on the classic Deal-Grove formulation [12]. This approach fails to account for the effects observed in $\mathrm{N}_{2} \mathrm{O}$ and $\mathrm{NO}$ grown/nitrided oxides, in particular the reduced growth rate that almost saturates with time. We have suggested neutralization of oxide growth sites by nitrogen as a possible cause for this effect [13], a hypothesis that is supported by the work of Tobin et al. [14]. This hypothesis is also consistent with the results that show reduced rate of

Manuscript received October 7, 1994; revised September 21, 1995. The review of this paper was arranged by Associate Editor A. H. Marshak. This model was developed for Technology Modeling Associates, Inc., Palo Alto, under TMA's University Partners Program. Some of the results presented in this paper appeared in TMA's publications, and were presented at different seminars as well as SPIE's Microelectronic Manufacturing '94 Conference.

The authors are with School of Microelectronic Engineering, Griffith University, Nathan, Brisbane, Queensland, 4111 Australia.

Publisher Item Identifier S 0018-9383(96)01078-7. interface-trap generation in $\mathrm{N}_{2} \mathrm{O}$ grown/nitrided oxides [3], $[4],[6]-[8]$, indicating that the nitrogen creates very strong bonds at the oxide-silicon interface which replace the weak bonds likely to generate interface traps.

This paper extends the Deal-Grove formulation to take into account the effects of growth-site neutralization. It is the aim of the paper to provide a better understanding of nitrogen modified oxide films, as well as to provide the basis for a growthkinetics model needed for process simulation. The process simulation aspect is discussed in terms of the models and approach used in TMA SUPREM-3 process simulator. [15]

\section{GROWTH KINETICS THEORY}

\section{A. Deal-Grove Formulation and TMA SUPREM-3 Oxidation Model}

The widely accepted Deal-Grove approach to oxide growth modeling [12] is based on the consideration of three consecutive fluxes of the oxidizing species: (i) flux due to diffusion of oxidizing species from the surface of the already grown oxide to the interface, $J_{S}$, (ii) flux due to chemical reaction of the oxidizing species with silicon at the interface, $J_{R}$, and (iii) flux due to the incorporation of the oxidizing species in the oxide, $J_{O}$. These fluxes are expressed as [12]:

$$
\begin{gathered}
J_{S}=\frac{D\left(C_{O}-C_{S}\right)}{x} \\
J_{R}=k_{r} C_{S} \\
J_{O}=C_{o x} \frac{d x}{d t}
\end{gathered}
$$

where $C_{O}, C_{S}$ and $C_{o x}$ are the concentrations of the oxidizing species at the surface of the oxide, at the interface and in the grown oxide, respectively, $D$ is the diffusion coefficient, $k_{r}$ is the interface reaction rate constant, and $x$ is the oxide thickness. Using that $J_{S}=J_{R}=J_{O}$ in the steady state, the following equation is obtained:

$$
\frac{d x}{d t}=\frac{C_{O}}{C_{o x}} \frac{D}{x+\frac{D}{k_{r}}}
$$

which can be expressed as

$$
\frac{d x}{d t}=\frac{B}{2 x+A}
$$

where $A=2 D / k_{r}$ and $B=2 D C_{O} / C_{o x}$.

The Deal-Grove model (5) predicts a linear oxide growth for $x \ll A / 2$, and a parabolic growth for $x \gg A / 2$. Accordingly, 
$B / A$ is referred to as the linear rate constant and $B$ as the parabolic rate constant. However, experimental results show that the linear oxide-growth model,

$$
\frac{d x}{d t}=\frac{B}{A} \equiv k_{r} \frac{C_{O}}{C_{o x}}
$$

underestimates the actual initial growth. As this effect cannot be neglected in sub- $20 \mathrm{~nm}$ oxides, and a generally acceptable theoretical approach has not been established, different empirical terms have been used to account for the so-called accelerated initial growth. In TMA SUPREM-3[15], for example, a growth rate constant $r_{t h i n}$ is added to account for the accelerated initial growth:

$$
\frac{d x}{d t}=\frac{B}{2 x+A}+r_{t h i n}
$$

The dependencies of the parabolic $(B)$, linear $(B / A)$ and the initial $\left(r_{\text {thin }}\right)$ growth rate constants on growth temperature $(T)$, pressure of the oxidizing gas $(P)$ and substrate doping concentration $(C)$ are given by [15]:

$$
B=\beta_{0} e^{-E_{A \beta} / k T} P^{\beta_{P}}\left(1+\delta_{0} e^{-E_{A \delta} / k T} C^{\eta_{0} \exp \left(-E_{A \eta} / k T\right)}\right)
$$

$$
\frac{B}{A}=l_{0} e^{-E_{A l} / k T} P^{l_{P}}\left(1+\gamma_{0} e^{-E_{A \gamma} / k T}\left(C_{V}-1\right)\right)
$$

and,

$$
r_{t h i n}=a_{0} e^{-E_{A a} / k T} e^{-x / x_{a}} P^{a_{P}} .
$$

$C_{V}$ in expr. (9) is the normalized vacancy concentration at the semiconductor surface, while all other symbols in exprs. (8) (10) represent different model parameters. It should be noted that some of the parameters have different values at higher and lower oxidizing temperatures.

The trend in semiconductor technology is toward the use of thinner oxides. In the case of sub-20nm oxides grown on a low-doped substrate at $P=1 \mathrm{~atm}$, the model used in TMA SUPREM-3 reduces to

$$
\frac{d x}{d t}=l_{0} e^{-E_{A l} / k T}+a_{0} e^{-x / x_{a}} e^{-E_{A a} / k T}
$$

where the first term represents the linear rate constant, while the second term is the initial growth constant.

\section{B. Extension of Deal-Grove Formulation}

It is assumed in the Deal-Grove model that the oxidation reaction at the interface is limited only by the concentration of oxidizing species at the interface $\left(C_{S}\right)$. Expression (2) of the Deal-Grove formulation clearly expresses this assumption. This excludes the possibility that the concentration of the oxide growth sites available at the interface influences the oxidation kinetics. In order to include the concentration of growth sites $\left(C_{g r}\right)$ as a possible factor in the oxidation reaction, we modify (2) as

$$
J_{R}=r C_{g r} C_{S}
$$

where $r$ is a reaction constant independent of the concentration of growth sites.

In oxides grown in $\mathrm{N}_{2} \mathrm{O}$, it becomes necessary to involve $C_{g r}$ in the oxidation model because there are two species involved, oxygen and nitrogen, that are competing for growth sites at the interface. This becomes especially obvious if it is assumed that the bonds that the nitrogen creates are much stronger than the strained oxygen bonds at the interface, which is seen as neutralization of the growth sites by the nitrogen atoms [13], [14]. The effect of oxidation rate reduction due to the incorporation of nitrogen in the growth sites, can only be modeled if the concentration of the growth sites $C_{g r}$ is included.

The introduced modification of expr. (2) also changes the equation for the linear rate constant (6), which now becomes:

$$
\frac{d x}{d t}=\frac{B}{A} \equiv r \frac{C_{O}}{C_{o x}} C_{g r}
$$

Introducing a new variable into the model, namely $C_{g r}$, means that a new equation is needed. We suggest that the continuity equation is applied to the oxide growth sites $C_{g r}$. Assuming that the growth sites exist only at the semiconductor-oxide interface, the continuity equation can be written as

$$
\frac{d C_{g r}}{d t}=G-R
$$

where $G$ and $R$ are the rates of generation and the recombination of the growth sites, respectively.

The assumption that the growth sites exist only at the semiconductor-oxide interface is taken to preserve the spirit of the Deal-Grove formulation which considers the interface reaction as the one that leads to oxide thickness increase. This is consistent with the recent experimental results by Gusev et al. [16], suggesting that the growth sites are basically suboxide states existing in the transition region between the silicon and the silicon-dioxide. This transition region is the "interface" between the silicon and the silicon-dioxide in the Deal-Grove terminology. Any possible diversity of chemical reactions inside the transition region is not considered in the Deal-Grove formulation which simply takes the overall effective reaction rate.

Remaining in the scope of developing a growth-kinetics model, any generation/recombination processes in the bulk of the oxide are ignored. This, however, by no means suggests that they do not exist. Take the example when the nitrogen is found only at the semiconductor-oxide interface, and not in the bulk of the oxide (this is generally the case of RTP grown oxides in $N_{2} O$ ). These results indicate that the generationrecombination processes in the bulk of the oxide lead to the liberation of nitrogen and replacement by oxygen, which is consistent with the fact that the silicon- nitrogen bond is weaker than the silicon-oxygen bond in the bulk of the dielectric (these data should not be confused with the strength of the bonds created by nitrogen at the interface when compared to the strained interface silicon-oxygen bonds). In a further development of the model, the continuity equation (14) can be extended to involve the bulk processes as well. In this paper, we consider the interface reaction only, to remain in the scope of the Deal-Grove formulation.

\section{Expression for the Concentration of Growth Sites}

An expression for the concentration of the growth sites can be obtained if (14) is solved. It is assumed that there is an Arrhenius-type dependence of the generation rate $G$ on 


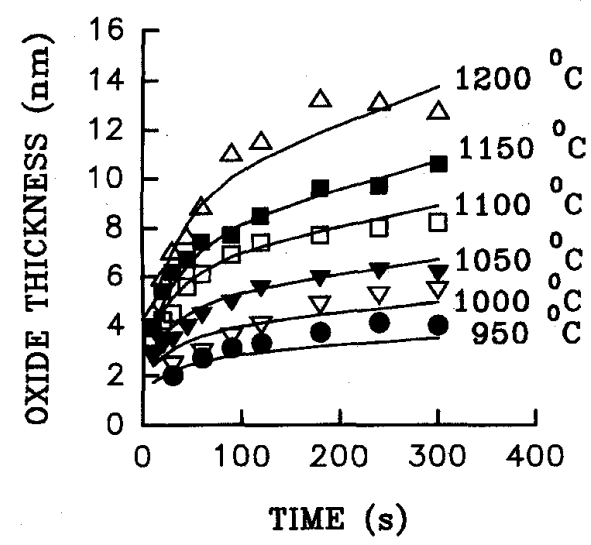

(a)
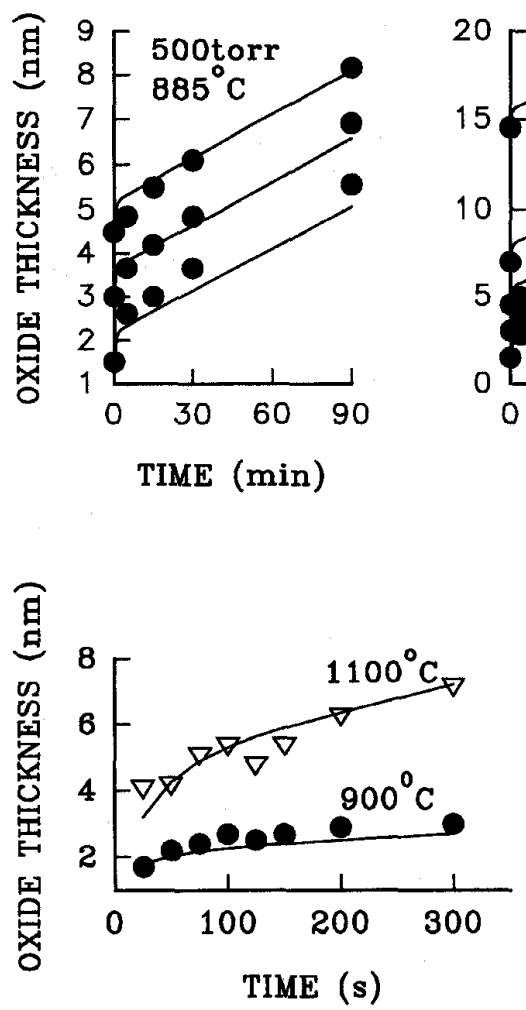

(d)

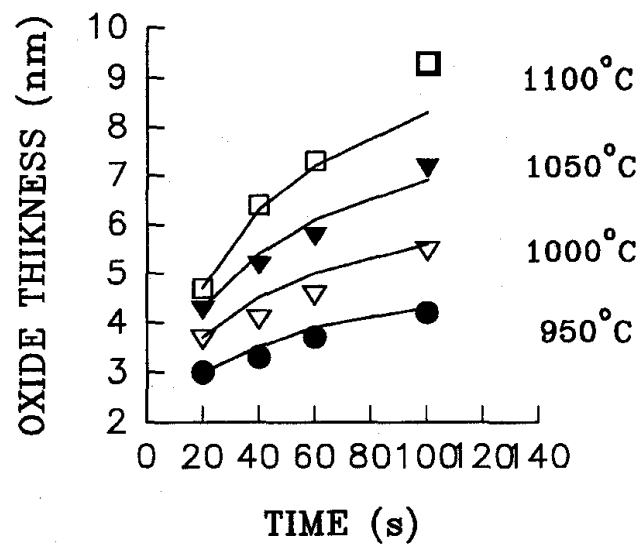

(b)

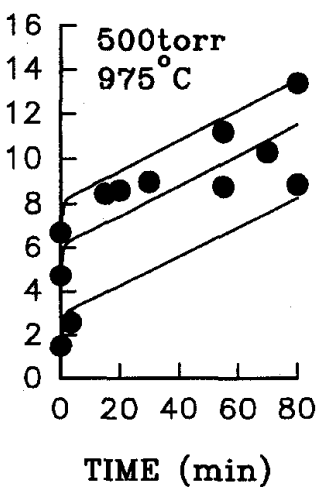

(c)

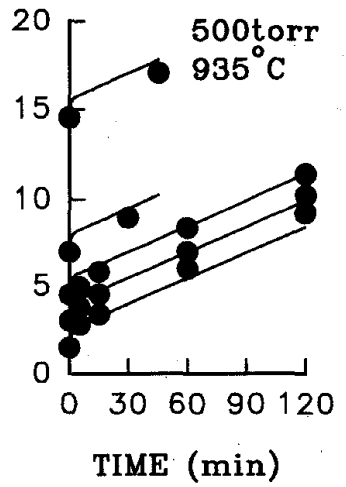

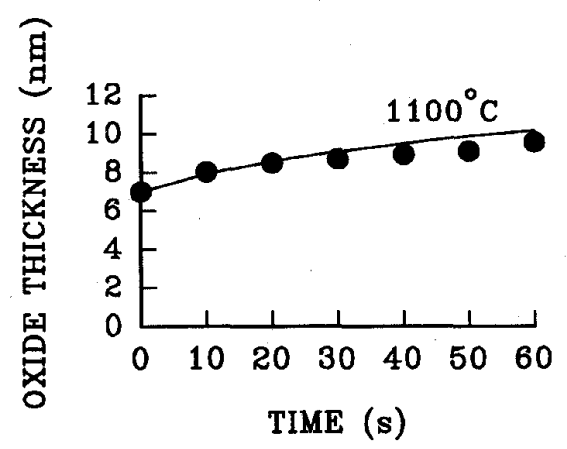

(e)

Fig. 1. Growth kinetics of oxides grown/nitrided in $N_{2} O$ on low-doped $\left.<100\right\rangle$ silicon substrates: lines - simulation with the new model; symbols - experimental data: (a) [18], (b) [19], (c) [17], (d) [20], and (e) [21].

temperature:

$$
G=G_{0} e^{-E_{A} / k T}
$$

where $E_{A}$ is the activation energy and $G_{0}$ is a temperature independent constant.

While the generation is driven by the temperature (thermal energy), the recombination appears due to existence of recombining species and sites to be recombined. Therefore, the recombination rate primarily depends on the concentration of the recombining species and the sites being recombined, while any temperature effects can be considered as secondorder effects. In this case, the recombining species are the oxygen, nitrogen and silicon, while the sites being recombined are the oxide growth sites $C_{g r}$. It is not necessary to involve the concentration of the recombining species in the model, as their concentration can be assumed to be constant during the oxidation process. This is not the case, however, with the growth sites, especially when it is assumed that they are being effectively neutralized by one of the recombining species. Therefore, the recombination rate can simply be expressed as

$$
R=\frac{1}{\tau} C_{g r}
$$

where $1 / \tau$ is a constant. 
Combining (14)-(16), the following differential equation is obtained:

$$
\frac{d C_{g r}}{d t}=G_{0} e^{-E_{A} / k T}-\frac{C_{g r}}{\tau} .
$$

The solution of (17) can be expressed as

$$
C_{g r}=\tau G_{0} e^{-E_{A} / k T}\left[1+\left(\frac{C_{g r}(0)}{\tau G_{0} \exp \left(-E_{A} / k T\right)}-1\right) e^{-t / \tau}\right]
$$

where $C_{g r}(0)$ is the concentration of growth sites at $t=0$.

In order to simplify expr. (18), it is assumed that $C_{g r}(0)$ follows the same temperature dependence as the generation rate $G$, thus $C_{g r}(0) \propto \exp \left(-E_{A} / k T\right)$. This assumption is correct as long as $C_{g r}(0)$ can be considered as independent of any neutralization of the growth sites by nitrogen. Effectively, this means that none of the generated growth sites at $t=0$ are immediately recombined (neutralized) by nitrogen. With this assumption, expr. (18) can be rewritten in a simpler form as:

$$
C_{g r}=l_{g r} e^{-E_{A} / k T}+a_{g r} e^{-t / \tau} e^{-E_{A} / k T},
$$

where $l_{g r}$ and $a_{g r}$ are time and temperature independent constants.

\section{TMA SUPREM-3 implementation}

For thin oxides, the approximation that $C_{0} \approx C_{S}$ is equivalent to the approximation $x \ll A / 2$ used to obtain the linear oxide-growth model. Using the thin oxide approximation $C_{0} \approx C_{S}$ along with the steady state condition $J_{S}=J_{R}=J_{0}$, (1), (3) and (12) lead to (13) for the growth rate of thin oxides. Using that the concentration of the growth sites in (13) is given by (19), the growth rate is obtained as:

$$
\frac{d x}{d t}=r \frac{C_{0}}{C_{o x}} l_{g r} e^{-E_{A} / k T}+r \frac{C_{0}}{C_{o x}} a_{g r} e^{-t / \tau} e^{-E_{A} / k T} .
$$

Expression (20) is very similar to (11), which is obtained from the model used in TMA SUPREM-3. The parameters can be transformed in the following way $l_{0}=r\left(C_{0} / C_{o x}\right) l_{g r}$, and $a_{0}=r\left(C_{0} / C_{o x}\right) a_{g r}$, which makes the similarity even more obvious:

$$
\frac{d x}{d t}=l_{0} e^{-E_{A} / k T}+a_{0} e^{-t / \tau} e^{-E_{A} / k T} .
$$

The first term in (21) is identical to the first, so-called linear rate term of (11). The only difference is in the second, so-called initial rate term, in that $e^{-x / x_{a}}$ used in TMA SUPREM-3 appears as $e^{-t / \tau}$ in (21). At first glance there appears to be no advantage in using the term $e^{-t / \tau}$ instead of the term $e^{-x / x_{a}}$, as the longer oxidation time $t$ corresponds to a larger oxide thickness $x$. This may well be correct for oxides continuously grown in one environment, however the situation changes when oxides initially grown in dry $\mathrm{O}_{2}$ to a certain thickness $x_{0}$ are further treated in an $N_{2} \mathrm{O}$ environment. If oxides with different initial oxide thicknesses $x_{0}$ are used, the difference between $e^{-x / x_{a}}$ and $e^{-t / \tau}$ terms becomes obvious. Namely, (11) predicts different growth rates due to different oxide thicknesses $x$, while (21) predicts the same growth rate during $\mathrm{N}_{2} \mathrm{O}$ oxidation, regardless of the differences in the thickness of the initially grown oxide $x_{0}$. The experimental data [17] confirm the prediction of the model with the $e^{-t / \tau}$ term.

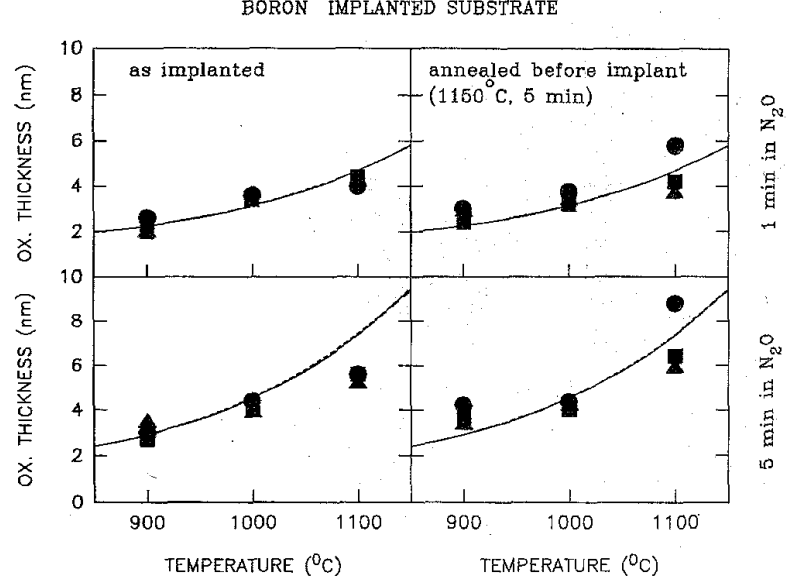

(a)

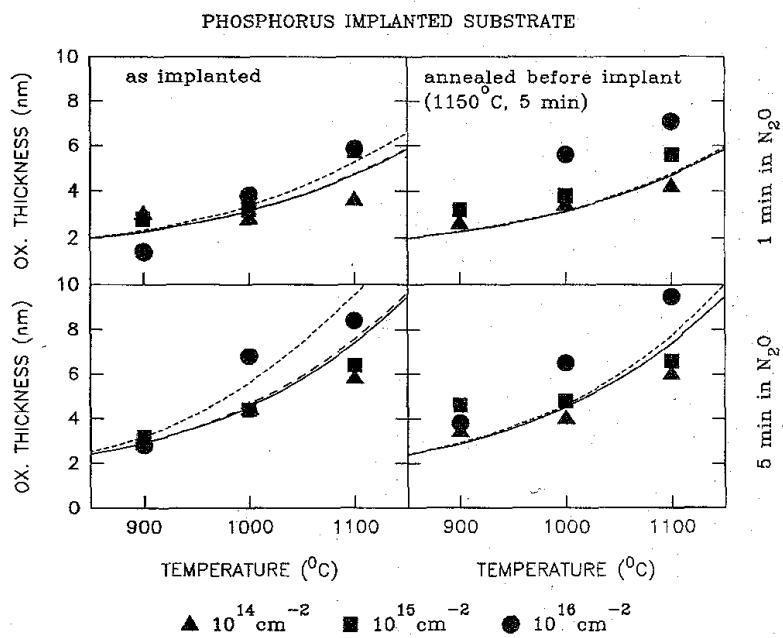

(b)

Fig. 2. Growth kinetics of oxides grown in $\mathrm{N}_{2} \mathrm{O}$ on implanted $<100>$ silicon substrates: lines - simulation with the new model; symbols - experimental data [9].

While the above discussion shows that the existing TMA SUPREM-3 model cannot be used for the oxides grown in $\mathrm{N}_{2} \mathrm{O}$, it also shows that the simplest adaptation can be made by replacing the variable $x$ in the initial rate term by the variable $t$. All other equations and terms in the oxidation rate model ((7)-(11)) can be retained. This is justified as the effects of substrate doping concentration and the pressure of the oxidizing gas, as well as the effect of parabolic growth rate in the diffusion-limited region, are qualitatively the same as in the case of oxidation in $\mathrm{O}_{2}$ or $\mathrm{H}_{2} \mathrm{O}$. Alike the situation where different values of model parameters are used for oxidation in $\mathrm{O}_{2}$ and $\mathrm{H}_{2} \mathrm{O}$, a new set of values of the model parameters have to be established for oxidation in $\mathrm{N}_{2} \mathrm{O}$.

\section{EXPERIMENTAL RESULTS AND MODEL PARAMETER EXTRACTION}

A number of experimental results on the growth kinetics for oxidation in $\mathrm{N}_{2} \mathrm{O}$ have appeared in the literature [17]-[21]. 


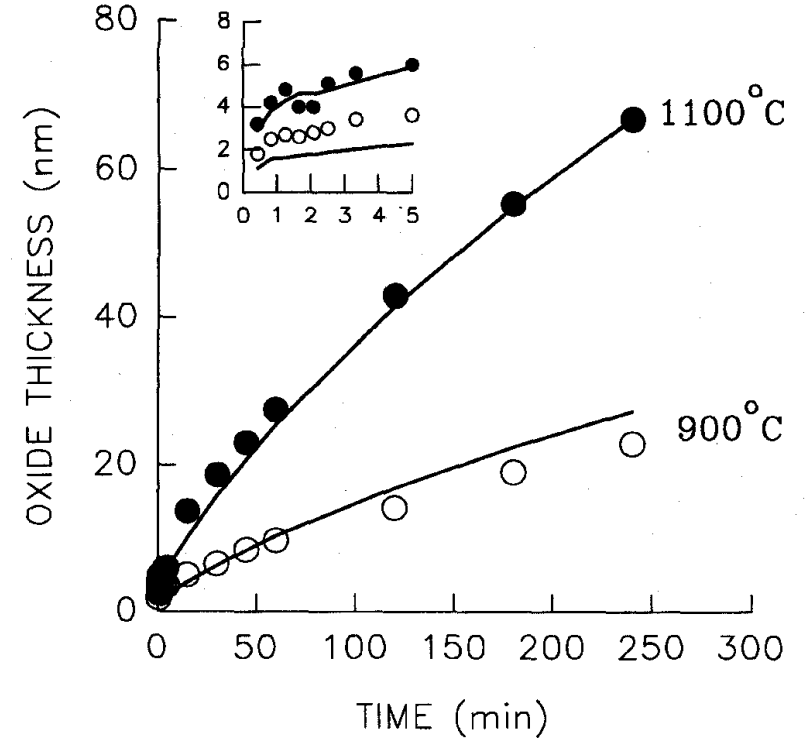

Fig. 3. Growth kinetics of oxides grown in $\mathrm{N}_{2} \mathrm{O}$ on low-doped $\langle 111\rangle$ silicon substrates: lines - simulation with the new model; symbols - experimental data [9], [22].

These results involve different oxidation times (from few seconds to $120 \mathrm{~m}$ ), different oxidation temperatures (from $885^{\circ} \mathrm{C}$ to $\left.1200^{\circ} \mathrm{C}\right)$, different pressures of the oxidizing gas $(1$ atm and 500 torr), and different initial oxide thicknesses (up to $15 \mathrm{~nm}$ ). All these results are, however, for oxides grown on low doped $<100>$ silicon. Sun et al. [22] published some experimental results for $\mathrm{N}_{2} \mathrm{O}$ oxides grown on $<111>$ low doped substrate, using very long times to show the parabolicgrowth effect. These results complement our data for low doped $<111>$ substrates [20], which were obtained with short oxidation times to emphasize the thin oxide regime. In addition, we obtained the first results on $\mathrm{N}_{2} \mathrm{O}$ growth kinetics using $<100>$ substrates implanted with boron and phosphorus with medium and high doses [9].

All these results were used to provide a unique and as comprehensive as possible set of parameters for the oxidation model described in the previous section. The values of the extracted parameters are given in Table I. The parameters that could not be extracted due to lack of experimental data are labeled as nc. This indicates that TMA SUPREM-3 default values for dry oxidation were assigned to the parameters labeled by nc when performing simulations of oxidation in $\mathrm{N}_{2} \mathrm{O}$. The simulation results (lines) are shown along with the experimental data (symbols) in Figs. 1-3, for oxidation on low-doped $\langle 100\rangle$ substrates, implanted $<100\rangle$ substrates, and low-doped $<111>$ substrates, respectively. It can be seen that a good agreement between the experimental data and the simulation using a single set of parameter values in TMA SUPREM-3 is achieved. The differences between the model and the experimental data appear to be well within measurement errors.

\section{SUMMARY AND CONCLUSIONS}

The continuing trend toward miniaturization of silicon devices is enforcing development of ultra-thin gate dielectrics.
TABLE I

Values of the Extracted Model Parameters for Oxidation in $\mathrm{N}_{2} \mathrm{O}$

\begin{tabular}{|c|c|c|c|c|}
\hline & \multicolumn{2}{|c|}{$<100>$} & \multicolumn{2}{|c|}{$<111>$} \\
\hline Symbol & Value & Unit & Value & Unit \\
\hline$a_{0}$ & 29.5 & $\mu m / m i n$ & 2.96 & $\mu m / \min$ \\
\hline$E_{A a}$ & 1.02 & $\mathrm{eV}$ & 0.655 & $e V$ \\
\hline$\tau$ & 0.617 & $\min$ & 0.304 & $\min$ \\
\hline$a_{P}$ & 0 & - & nc & - \\
\hline$l_{0}$ & 2.8 & $\mu m / \min$ & 0.127 & $\mu m / \min$ \\
\hline$E_{A l}$ & 1.02 & $e V$ & 0.655 & $e V$ \\
\hline$l_{P}$ & 2.8 & - & nc & - \\
\hline$\gamma_{0}$ & 1588 & - & $\mathrm{nc}$ & - \\
\hline$E_{A \gamma}$ & 1.295 & $\mathrm{eV}$ & nc & - \\
\hline$\beta_{0}$ & $\mathrm{nc}$ & - & 0.931 & $\mu m^{2} / \min$ \\
\hline$E_{A \beta}$ & nc & - & 1.1932 & $e V$ \\
\hline$\beta_{P}$ & nc & - & nc & - \\
\hline$\delta_{0}$ & nc & - & nc & - \\
\hline$E_{A \delta}$ & $\mathrm{nc}$ & - & nc & - \\
\hline$\eta_{0}$ & $\mathrm{nc}$ & - & nc & - \\
\hline$E_{A \eta}$ & $\mathrm{nc}$ & - & nc & $=$ \\
\hline
\end{tabular}

While thermally grown $\mathrm{SiO}_{2}$ has been used as a gate dielectric ever since the era of silicon devices began, it appears that the electrical and physical properties of pure $\mathrm{SiO}_{2}$ are not good enough to provide acceptable ultra-thin gate dielectrics. It has been found, however, that oxides grown or nitrided in $\mathrm{N}_{2} \mathrm{O}$ exhibit significantly improved electrical and physical properties. The growth kinetics of these oxides is dramatically different to the kinetics of oxides grown in dry $\mathrm{O}_{2}$ or $\mathrm{H}_{2} \mathrm{O}$. The currently used models for growth kinetics, based on classic Deal-Grove formulation, fail to account for the effects observed in $\mathrm{N}_{2} \mathrm{O}$ grown/nitrided oxides, in particular reduced, almost saturating growth rate.

It is assumed in the Deal-Grove model that the oxidation reaction at the interface is limited only by the concentration of oxidizing species at the interface. This excludes the possibility that the concentration of the growth sites available at the interface may influence the oxidation process. In the oxides grown in $\mathrm{N}_{2} \mathrm{O}$, however, the nitrogen species "neutralize" the growth sites which slows down the oxidation reaction. 
In this paper, the Deal-Grove formulation has been extended to include the concentration of the growth sites. Also, the continuity equation applied to the growth sites has been used to determine the concentration of the growth sites. This model has been incorporated into TMA SUPREM-3 process simulator, and the model parameters calibrated with the experimental data available. This provides not only a needed tool for process simulation, but also a better understanding of nitrogen modified oxide films.

\section{ACKNOWLEDGMENT}

The authors would like to acknowledge TMA for providing TMA SUPREM-3 simulator and for helpful discussions.

\section{REFERENCES}

[1] D. Kahng, "A historical perspective on the development of MOS transistors and related devices," IEEE Trans.-Electron Devices, vol. ED-23, p. 655, 1976.

[2] H.B. Harrison, and S. Dimitrijev , "Ultra-thin dielectrics for semiconductor applications - growth and characteristics," Microelectron. $J$, vol. 22, p. 3, 1991

[3] A. Uchiyama, H. Fukuda, T. Hayashi, T. Iwabuchi, and S. Ohno, "High performance $\mathrm{P}^{+}$-gate $\mathrm{P}$ MOSFET's with $\mathrm{N}_{2} \mathrm{O}$ nitrided $\mathrm{SiO}_{2}$ gate films," Electron. Lett.., vol. 26, p. 1932, 1990.

[4] H. Fukuda, T. Arakawa, and S. Ohno, "Highly reliable thin nitrided $\mathrm{SiO}_{2}$ films formed by rapid thermal processing in $\mathrm{N}_{2} \mathrm{O}$ ambient," Electron. Lett., vol. 26, p. 1505, 1990.

[5] G.Q. Lo, W. Ting, J. Ahn, and D.L. Kwong, "P-channel MOSFET's with ultrathin $\mathrm{N}_{2} \mathrm{O}$ oxides," IEEE Electron Device Lett., vol. 13, p. 111, 1992.

[6] A.B. Joshi, G. Yoon, J. Kim, G.Q. Lo, and D.-L. Kwong, "Highfield breakdown in thin oxides grown in $\mathrm{N}_{2} \mathrm{O}$ ambient," IEEE Trans. Electron Devices., vol. 40, p. 1437, 1993.

[7] G.Q. Lo, A.B. Joshi, and D.L. Kwong, "Radiation hardness of MOSFET's with $\mathrm{N}_{2} \mathrm{O}$-nitrided gate oxides," IEEE Trans. Electron Devices., vol. 40 , p. $1565,1993$.

[8] Z. Liu, H.-J. Wann, P.K. Ko, C. Hu, and Y.C. Cheng,' 'Effects of $\mathrm{N}_{2} \mathrm{O}$ anneal and reoxidation on thermal oxide characteristics," IEEE Electron Device Lett., vol. 13, p. 402, 1992.

[9] H. B. Harrison, A. Misiura, S. Dimitrijev, D. Sweatman, Z.-Q. Yao and Y.-T. Yeow, "Dielectrics on silicon thermally grown or annealed in a nitrogen rich environment,"' in Rapid Thermal and Integrated Processing II, J.J. Wortman, J.C. Gelpey, M.L. Green, S.R.J. Brueck, and F. Roozeboom, Eds., MRS Symp. vol. 342, pp. 151-161, 1994.

[10] Z.-Q. Yao, H. B. Harrison, S. Dimitrijev, D. Sweatman and Y. T. Yeow, "High quality ultrathin dielectric films grown on silicon in a nitric oxide ambient," Appl. Phys. Lett., vol. 64, no. 26, pp. 3584-3586, 1994.

[11] Z.-Q. Yao, H. B. Harrison, S. Dimitrijev and Y.-T. Yeow, "The electrical properties of sub-5nm oxynitride dielectrics prepared in a nitric oxide ambient using rapid thermal processing," IEEE Electron Device Lett., vol. 15, pp. 516-518, 1994

[12] B. E. Deal and A. S. Grove, "General relationship for the thermal oxidation of silicon;" J. Appl. Phys., vol. 36, p. 3770, 1965.

[13] S. Dimitrijev, D. Sweatman, H. B. Harrison, "Model for dielectric growth on silicon in a nitrous oxide environment," Appl. Phys. Lett., vol. 62,13 , pp. 1539-1540, 1993.

[14] P. J. Tobin, Y. Okada, S. A. Ajuria, V. Lakhotia, W.A. Feil and R.I. Hedge, "Furnace formation of silicon oxynitride thin dielectrics in nitrous oxide $\left(\mathrm{N}_{2} \mathrm{O}\right)$ : The role of nitric oxide (NO)," J. Appl. Phys., vol. 75 , no. 3 , pp. 1811-1817, 1994.

[15] TMA SUPREM-3, One-Dimensional Semiconductor Process Simulation Technology Modeling Associates, 1993 (vol. 1, p. 2-52).

[16] E.P. Gusev, H.C. Lu, T. Gustafsson, and E. Garfunkel, "Growth mechanism of thin silicon oxide films on $\mathrm{Si}(100)$ studied by mediumenergy ion scattering,"' Phys. Rev. B, vol. 52, 15 July 1995.

[17] H.R. Solemni, A. Philipossian and B. Doyle, "A study of the growth kinetics of $\mathrm{SiO}_{2}$ in $\mathrm{N}_{2} \mathrm{O}$," in IEDM Tech. Dig., 1992, pp. 629-632.
[18] W. Ting, H. Hwang, J. Lee and D.L. Kwong, "Growth Kinetics of Ultrathin $\mathrm{SiO}_{2}$ Films Fabricated by Rapid Thermal Oxidation of $\mathrm{Si}$ Substrates in $\mathrm{N}_{2} \mathrm{O}$," J. Appl. Phys., vol. 70, 2, pp. 1072-1074, 1991.

[19] G.W. Yoon, A.B. Joshi, J. Ahn and D.L. Kwong, "Thickness uniformity and electrical properties of ultrathin gate oxides grown in $\mathrm{N}_{2} \mathrm{O}$ ambient by Rapid Thermal Processing,"' $J$. Appl. Phys., vol. 72, no. 12, pp. 5706-5710, 1992.

[20] H.B. Harrison, S. Dimitrijev, D. Sweatman, A. Misiura and G.K. Reeves, "Substrate doping and orientation effects on dielectric growth on silicon in a nitrous oxide environment," in Rapid Thermal and Integrated Processing II, J.C. Gelpey, J.K. Elliot, J.J. Wortman and A. Ajmera, Eds., MRS Symp. Proc. vol. 303, pp. 417-420 (1993).

[21] N. Bellafiore, F. Pio and C. Riva, "Thin oxide nitridation in $\mathrm{N}_{2} \mathrm{O}$ by RTP for non-volatile memories," Microelec. J., vol. 24, pp. 453-458, 1993.

[22] S.C. Sun and H.Y. Chang, "Oxidation simulation and growth kinetics of thin $\mathrm{SiO}_{2}$ in pure $\mathrm{N}_{2} \mathrm{O}$," in Simulation of Semiconductor Devices and Processes, vol. 5, S. Selberherr, H. Stippel, and E. Strasser, Ed., pp. 169-172, 1993.

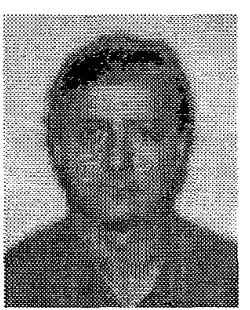

Sima Dimitrijev (S'87-M'88) received B.Eng., M.Sc., and Ph.D. degrees in electronic engineering from the University of Nis, Yugoslavia, in 1982, 1985 , and 1989 , respectively.

During 1982 and 1983, he was with the Semiconductor Factory of the Electronics Industry, $\mathrm{Nis}$, where he worked on the development of CMOS technology. From 1983 to 1990 , he was with the Faculty of Electronic Engineering at the University of Nis. In 1990, he joined Griffith University, Brisbane, Australia, as a Lecturer and later Senior Lecturer at the School of Microelectronic Engineering. His current research interests are in the fields of novel silicon devices and ultra-thin gate dielectrics. He has published over 80 papers in the technical literature on MOSFET instabilities, IC yield modeling, MOSFET modeling, ultra-thin gate dielectrics, and deep- submicron manufacturing issues. He is currently developing a novel FET for power-supply applications.

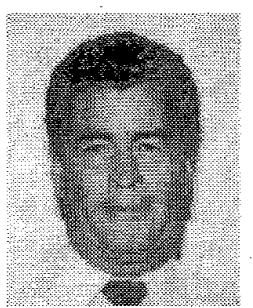

H. Barry Harrison is currently Head of School of Microelectronic Engineering and Chairman of the Engineering Board of Studies at Griffith University. Professor Harrison is technical consultant on the Board of Microelectronic Technologies Pty Ltd (a company that was established due to his incentive) and a Director of the Space Centre for Microelectronic Technology. He is also a consultant to other companies on microelectronic related matters.

He has written in excess of 200 papers, one book and two book chapters. His research interests are in the area of limits to scale of integration, contact modeling and semi-custom I.C. architecture

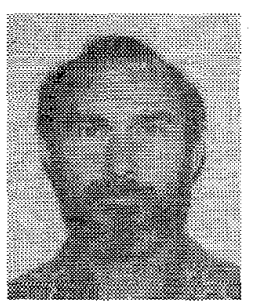

Denis Sweatman obtained the B.Sc.(Hons) degree in 1972, and the Ph.D. degree in 1982 in physical chemistry (spectroscopy). from the University of Queensland, Austalia.

From 1982 to 1985 , he researched and developed laser systems at the Laser Application Laboratory at the University of Queensland. From 1986 to 1991; he was R\&D Scientist at a scientific instrument manufacturer in Queensland (Ionode P/L). Since 1991, he has been involved in research and commissioning of the Fabrication Laboratory at Griffith University and is now a Lecturer at the School of Microelectronic Engineering. He is currently investigating ultra thin gate dielectrics on silicon and sensors. 